\title{
Video Article \\ An Inertial Measurement Unit Based Method to Estimate Hip and Knee Joint Kinematics in Team Sport Athletes on the Field
}

\author{
Bram J.C. Bastiaansen* ${ }^{*}$, Erik Wilmes ${ }^{2}$, Michel S. Brink ${ }^{1}$, Cornelis J. de Ruiter ${ }^{2}$, Geert J.P. Savelsbergh ${ }^{2}$, Annemarijn Steijlen ${ }^{3}$, Kaspar \\ M.B. Jansen ${ }^{3}$, Frans C.T. van der Helm ${ }^{4}$, Edwin A. Goedhart ${ }^{5}$, Doris van der Laan ${ }^{6}$, Riemer J.K. Vegter ${ }^{1}$, Koen A.P.M. Lemmink ${ }^{1}$ \\ ${ }^{1}$ Center for Human Movement Sciences, University Medical Center, University of Groningen \\ ${ }^{2}$ Department of Human Movement Sciences, Faculty of Behavioural and Movement Sciences, Vrije Universiteit Amsterdam \\ ${ }^{3}$ Emerging Materials, Department of Design Engineering, Delft University of Technology \\ ${ }^{4}$ Department of Biomechanical Engineering, Delft University of Technology \\ ${ }^{5}$ FIFA Medical Center, Royal Netherlands Football Association \\ ${ }^{6}$ Royal Dutch Hockey Association \\ * These authors contributed equally
}

Correspondence to: Bram J.C. Bastiaansen at b.j.c.bastiaansen@umcg.nl

URL: https://www.jove.com/video/60857

DOI: doi:10.3791/60857

Keywords: Behavior, Issue 159, inertial measurement units, athletes, soccer, hockey, lower extremity, kinematics, injury prevention, performance enhancement, smart textiles, running

Date Published: 5/26/2020

Citation: Bastiaansen, B.J., Wilmes, E., Brink, M.S., de Ruiter, C.J., Savelsbergh, G.J., Steijlen, A., Jansen, K.M., van der Helm, F.C., Goedhart, E.A., van der Laan, D., Vegter, R.J., Lemmink, K.A. An Inertial Measurement Unit Based Method to Estimate Hip and Knee Joint Kinematics in Team Sport Athletes on the Field. J. Vis. Exp. (159), e60857, doi:10.3791/60857 (2020).

\section{Abstract}

Current athlete monitoring practice in team sports is mainly based on positional data measured by global positioning or local positioning systems. The disadvantage of these measurement systems is that they do not register lower extremity kinematics, which could be a useful measure for identifying injury-risk factors. Rapid development in sensor technology may overcome the limitations of the current measurement systems. With inertial measurement units (IMUs) securely fixed to body segments, sensor fusion algorithms and a biomechanical model, joint kinematics could be estimated. The main purpose of this article is to demonstrate a sensor setup for estimating hip and knee joint kinematics of team sport athletes in the field. Five male subjects (age $22.5 \pm 2.1$ years; body mass $77.0 \pm 3.8 \mathrm{~kg}$; height $184.3 \pm 5.2 \mathrm{~cm}$; training experience $15.3 \pm 4.8$ years) performed a maximal 30-meter linear sprint. Hip and knee joint angles and angular velocities were obtained by five IMUs placed on the pelvis, both thighs and both shanks. Hip angles ranged from $195^{\circ}\left( \pm 8^{\circ}\right)$ extension to $100.5^{\circ}\left( \pm 8^{\circ}\right)$ flexion and knee angles ranged from $168.6^{\circ}$ $\left( \pm 12^{\circ}\right)$ minimal flexion and $62.8^{\circ}\left( \pm 12^{\circ}\right)$ maximal flexion. Furthermore, hip angular velocity ranged between $802.6^{\circ} \cdot \mathrm{s}^{-1}\left( \pm 192^{\circ} \cdot \mathrm{s}^{-1}\right)$ and $-674.9^{\circ}$ ${ }^{\circ} \cdot \mathrm{s}^{-1}\left( \pm 130^{\circ} \cdot \mathrm{s}^{-1}\right)$. Knee angular velocity ranged between $1155.9^{\circ} \cdot \mathrm{s}^{-1}\left( \pm 200^{\circ} \cdot \mathrm{s}^{-1}\right)$ and $-1208.2^{\circ} \cdot \mathrm{s}^{-1}\left( \pm 264^{\circ} \cdot \mathrm{s}^{-1}\right)$. The sensor setup has been validated and could provide additional information with regard to athlete monitoring in the field. This may help professionals in a daily sports setting to evaluate their training programs, aiming to reduce injury and optimize performance.

\section{Video Link}

The video component of this article can be found at https://www.jove.com/video/60857/

\section{Introduction}

Team sports (e.g., soccer and field hockey) are characterized by alternating brief explosive actions such as high-intensity running or sprinting, with longer periods of less demanding activities like walking or jogging ${ }^{1,2,3,4,5,6}$. Over the last decades, the physical demands of the game evolved with more distance covered at high speed and sprinting, faster ball speeds and more passes ${ }^{7,8}$.

Athletes constantly train hard in order to maintain and improve their physical capacity to withstand the physical demands of the game. The correct application of a training stimulus in combination with sufficient recovery induces responses that lead to adaptation of the human body, improving fitness and performance ${ }^{9}$. On the contrary, an imbalance between a training stimulus and recovery can lead to prolonged fatigue and an undesirable training response (maladaptation), which increases the risk of injury in both professional and amateur team sport athletes $^{10,11,12,13}$.

One of the major risks accompanied with high training and match stimuli are muscle strain injuries. Muscle strain injuries constitute more than a third of all time-loss injuries in team sports and cause more than a quarter of the total injury absence, with the hamstrings being the most frequently involved ${ }^{14,15,16,17}$. Furthermore, the number of athletes that sustain a hamstring strain injury rises each year ${ }^{18,19}$, despite that multiple programs have been introduced to prevent hamstring strain injuries ${ }^{12,13,20,21}$. Consequently, this has a negative influence from sportive ${ }^{22}$ and 
financial ${ }^{23}$ perspectives. Thus, adequate monitoring of individual athletes is essential for optimizing training schedules, minimizing injury risk and optimizing performance.

Current athlete monitoring practice in team sports is mainly based on position data measured by local or global positioning systems ${ }^{24,25}$. These systems monitor activity with GPS-based metrics such as distance covered, average running velocity or accelerometry-based metrics such as PlayerLoad ${ }^{26,27,28}$. A disadvantage of these measures is that they do not include lower extremity kinematics. Optoelectronic measurement systems serve as a gold standard to perform a kinematic analysis of the lower extremities during a linear sprint ${ }^{29,30,31,32}$. The disadvantages of these systems are a lack of ecological validity due to their restricted measurement area, the need of an expert to operate the system and the time consuming data analysis. Thus, this method is not suitable for daily sports practice.

Rapid development in sensor technology may overcome the limitations of current methods to monitor athletes. The recent reliability, miniaturisation and data storage possibilities of inertial measurement units (IMU) enables in-field application of sensor technology. IMUs contain an accelerometer, gyroscope and magnetometer which measure the acceleration, angular velocity and magnetic field, in three orthogonal axes respectively ${ }^{33,34}$. With sensors securely fixed to body segments, sensor fusion algorithms and a biomechanical model, it is possible to estimate joint kinematics ${ }^{33}$. The registration of joint kinematics in combination with information about the acceleration of different body segments may improve athlete monitoring in team sports.

By coupling the IMU sensor setup to a standardized field test, it can be illustrated how lower extremity kinematics are registered during linear sprinting in the field, which could be a useful measure for identifying injury-risk factors. The sensor setup could provide additional information to current monitoring measures that professionals may use for optimizing training schedules to improve performance and minimize injury risk. Therefore, the main purpose of this article is to demonstrate an inertial sensor setup for estimating hip and knee joint kinematics of team sport athletes in the field.

\section{Protocol}

All methods described in this section have been approved by the ethical committee of the Center for Human Movement Sciences of the University of Groningen (Register number: 201800904).

\section{Field test and inertial measurement unit preparation}

1. Set two cones at least $1 \mathrm{~m}$ from each other to determine the start of the field test. NOTE: The 1-m distance between the cones enables the subject to run easily through the starting point of the field test. This distance can be adjusted to the preference of the test leader.

2. Determine the end point of the field test by rolling measuring tape from the starting point of the test until a 30 -m linear distance has been travelled.

3. Set two cones at least $1 \mathrm{~m}$ from each other to determine the end point of the field test.

4. Prepare the IMUs to get properly attached to the subject's body. NOTE: See Table of Materials for IMU dimensions and weight characteristics.

1. Cut stretch tape into 5 pieces the size of $10 \mathrm{~cm} \times 10 \mathrm{~cm}$.

2. Cut double-sided adhesive tape (e.g., toupee tape) into 5 pieces equal to the size of the IMUs that are used.

3. Attach a piece of double-sided adhesive tape to each IMU.

4. Label each IMU, so that it can be recognized individually during data analysis.

\section{Subject preparation}

1. Obtain information about the subject's gender, age, body weight, and height. Ask the subject to fill in a questionnaire about their background in team sports. Obtain written informed consent from subjects that meet the inclusion criteria.

NOTE: Examples of questions: (i) For how many years do you play soccer? (ii) At which level do you play soccer? (iii) How many hours per week do you have soccer training during the past 6 months? (iv) What is your playing position? (v) Did you experience any pain or did you sustain a musculoskeletal injury at the lower extremity during the past 6 months?

2. Determine if the subject meets the inclusion criteria.

NOTE: Include subjects when they did not experience any musculoskeletal injuries or pain in the lower extremities in the 6 months before executing the protocol; Subjects should have more than 1-year experience in competing team sports at amateur level.

3. Ask the subject to change into sports clothing (e.g., a soccer shirt, soccer shorts and soccer shoes). NOTE: Because sensors will be placed on the tibia, soccer socks are unwanted.

4. Prepare the IMUs for attaching to the subject's body.

1. Align all 5 IMUs next to each other.

2. Activate all five IMUs at the same time by pushing a button on top of the sensor. The sensor is activated when a green light is blinking. NOTE: From this moment, each IMU samples data at $500 \mathrm{~Hz}$. Data is stored on a SD card internally. Data has to be uploaded to a laptop or computer after the test has been completed.

3. Ensure that a mechanical peak has been generated by tapping all IMUs on a hard surface at the same time (e.g., on a table). NOTE: The mechanical peak is needed for synchronizing the IMU signals. Synchronization of the IMU signals is performed during data processing (section 5). This section is not necessary when commercially available sensors are used. In that case, use the corresponding software to synchronize the sensors.

5. Attach the IMUs to the subject's body (Figure 1). 
1. Shave the subject's body hair at the following anatomical locations: at the sacrum between both posterior superior iliac spines, the anteromedial bony part of both right and left tibia, and the lateral part of both right and left thigh (i.e., tractus illiotibialis). NOTE: The anatomical locations where sensors should be placed can be determined by palpation.

2. Spray adhesive spray on the anatomical locations described in step 2.5.1. Wait 5-10 s to make sure the adhesive spray is dry. NOTE: Hold the spray at least $10 \mathrm{~cm}$ (4 inches) away from the skin and spray the desired area with a sweeping motion.

3. Remove the protective layer of the double-sided adhesive tape from the IMUs.

4. Place the IMU at the described anatomical locations. Write down the anatomical location with the corresponding label of the IMU (e.g., right shank: IMU 1).

5. Attach the stretching tape on top of each IMU to make sure the sensor is additionally secured to the skin.

\section{IMU sensor calibration}

1. Instruct the subject to stand still in a neutral position with their feet hip-width apart and their hands in their side. Maintain this position for a minimum period of $5 \mathrm{~s}$.

2. Instruct the subject to flex their left hip and knee to a $90^{\circ}$ angle in the sagittal plane followed by extending the hip to their neutral position as described in step 3.1.

NOTE: For definitions of kinematic variables, see Figure 2.

3. Repeat step 3.2 for the right hip and knee.

4. Instruct the participant to flex their trunk towards the ground and return to their neutral position

5. Wait for a minimum period of $5 \mathrm{~s}$.

6. Repeat steps 3.1-3.5 once.

\section{Execute the 30-m linear sprint test}

1. Instruct the subject to perform a warming up (e.g., the soccer specific FIFA $11^{+}$warming up programme ${ }^{20}$ ).

2. Inform the subject about the test protocol.

1. Clearly state that the risk of sustaining an injury during the test is not higher than during normal training and that the subject can abort the test at any time, without giving any reason.

2. Instruct the subject to stand in the correct starting position, with their preferred foot standing on the starting line and their shoulders behind the starting line on the field.

3. Instruct the subject that the test leader will count down from 3 to 0 followed by shouting 'Start'. Instruct that when 'Start' has been called, the test begins.

4. Inform the subject to sprint as fast as possible until the 30-m end point has been reached. After the 30-m finish point has been reached, the subject has to decelerate as fast as possible to a standstill position.

5. Allow the subject to ask questions. If needed, allow the subject to perform a practice run to familiarize the subject with the protocol.

3. Ask the subject if the instructions were clear.

4. Make sure the subject is in the correct starting position.

5. Count down from ' 3 ' to ' 0 ' and shout 'Start' to start the test. Start the timer when the start sign has been given.

6. Encourage the subject in order to achieve maximal performance.

7. Stop the timer when the participant has reached their standstill position.

8. Repeat steps 4.4-4.6 until three sprints have been performed.

NOTE: Let participants rest for at least 2 min between trials. Include the fastest sprint for data analysis.

9. Instruct the subject to perform a cooling down.

10. Detach the IMUs from the subject by removing the stretch tape and double-sided adhesive tape from the subject's body.

\section{Data processing}

1. Connect the IMU with a computer by using a USB cable. Export the raw IMU data to a specific folder on the computer.

2. Open MATLAB (version r2018b). Import the raw IMU data files (i.e., accelerometer, gyroscope and magnetometer data).

3. Synchronize the raw IMU data files.

1. Import the acceleration data file of one sensor (e.g., pelvis sensor).

2. Calculate jerk by differentiating the $X, Y$ and $Z$ acceleration signals. Sum up the $X, Y$, and $Z$ jerk to obtain the total jerk.

3. Obtain the mechanical peak by finding the index value in the data file where the total jerk has reached its maximal value. The index value is the start of the measurement.

4. Delete all data points of the acceleration data, magnetometer data and gyroscope data before the index value of the sensor.

5. Repeat steps 5.3.1-5.3.3 for each raw data file of the corresponding sensor.

6. Determine which sensor contains the lowest amount of data points by obtaining the number of sampled data points for each data file.

7. Cut all other data files equal to the size of the sensor that registered signals for the shortest time period.

4. Filter gyroscope data using a second order low-pass Butterworth filter with a cutoff frequency of $12 \mathrm{~Hz}$.

NOTE: The filter and particular cutoff frequency were chosen based on visual data inspection in previous pilot experiments.

5. Obtain sensor orientation with respect to the global earth frame by calculating the orientation quaternion of the sensor using a Madgwick filter $^{35}$

NOTE: An extensive description of how sensor orientation with respect to the global earth frame is calculated is described in Madgwick et al. $^{35}$.

6. Align the sensor coordinate frame to body segment. 
1. Select the index numbers of the data file when the subject was standing still during calibration (step 3.1).

NOTE: It is assumed that the longitudinal axis of the sensor is similar to the gravity vector.

2. Use the index numbers of step 5.6.1 to calculate the average orientation of each sensor with respect to the global reference frame during the static calibration. Then rotate the sensor frame of each sensor, so that it aligns with the global reference frame during static calibration.

3. Select the index numbers of the data file when the calibration movement of the left leg was performed (step 3.2).

4. Rotate the orientation of the left leg sensors in such a way that the calibration movement is a rotation about the frontal axis only.

5. Repeat steps 5.6.3 and 5.6.4 for the calibration movements of the right leg and trunk.

7. Obtain joint orientations by expressing the orientation of the distal body segment in the coordinate frame of the proximal segment for each joint.

8. Obtain joint angles by decomposing the obtained joint orientations into ' $X Z Y$ ' Euler angles. NOTE: How to decompose the obtained joint orientations into ' $X Z Y$ ' Euler angles is described in the work of Diebel ${ }^{36}$

9. Obtain joint angular velocities expressing the gyroscope signals of each distal segment in the coordinate frame of its corresponding proximal segment minus the angular velocity of the proximal segment.

10. Identify each step during the linear sprint by using a step detection algorithm.

1. Import the filtered gyroscope data in MATLAB.

2. Use a peak detection function to identify peaks in the gyroscope signal. NOTE: Peak height was set at $286.5^{\circ} \cdot \mathrm{s}^{-1}$ and minimal peak distance was set at 100 samples $(=0.2 \mathrm{~s})$.

11. For each step, calculate the maximum value for hip angle, knee angle, hip angular velocity, and knee angular velocity.

12. For each step, calculate the minimum value for hip angle, knee angle, hip angular velocity, and knee angular velocity.

13. For each step, calculate the hip range of motion by subtracting the minimum hip angle from the maximum hip angle.

14. For each step, calculate the knee range of motion by subtracting the minimum knee angle from the maximum knee angle.

15. Save the processed data to a specific folder on the computer in order to use it for further analysis.

\section{Data analysis}

1. Import the processed IMU data in MATLAB.

2. Divide the sprint into an acceleration, a top speed and deceleration phase based on the steps identified by the step detection algorithm. NOTE: Sprint phases in this article were chosen arbitrarily. The acceleration phase is defined as step 3 to $8^{37}$, whereas the deceleration phase is defined as the last eight steps of the sprint. Top speed data was derived from steps performed between these phases.

3. Select the angular velocity data for data analysis.

4. Calculate the mean values and standard deviation of the kinematic variables of all steps during each phase of the 30-m linear sprint test.

5. Repeat step 6.3 and 6.4 for the angle data

\section{Representative Results}

Five subjects (all male; all soccer players; age $22.5 \pm 2.1$ years; body mass $77.0 \pm 3.8 \mathrm{~kg}$; height $184.3 \pm 5.2 \mathrm{~cm}$; training experience $15.3 \pm 4.8$ years) performed a maximal 30-m linear sprint. Hip angles ranged between $100.5^{\circ}\left( \pm 8^{\circ}\right)$ maximal flexion and $183.1^{\circ}\left( \pm 8^{\circ}\right)$ maximal extension during acceleration, $104.1^{\circ}\left( \pm 8^{\circ}\right)$ maximal flexion and $195^{\circ}\left( \pm 8^{\circ}\right)$ maximal extension during top speed, and $128.4^{\circ}\left( \pm 11^{\circ}\right)$ maximal flexion and $171.9^{\circ}\left( \pm 23^{\circ}\right)$ minimal flexion during deceleration. Hip angular velocities ranged between $744.9^{\circ} \cdot \mathrm{s}^{-1}\left( \pm 154^{\circ} \cdot \mathrm{s}^{-1}\right)$ and $-578^{\circ} \cdot \mathrm{s}^{-1}\left( \pm 99^{\circ} \cdot \mathrm{s}^{-1}\right)$ during acceleration, $802.6^{\circ} \cdot \mathrm{s}^{-1}\left( \pm 192^{\circ} \cdot \mathrm{s}^{-1}\right)$ and $-674.9^{\circ} \cdot \mathrm{s}^{-1}\left( \pm 130^{\circ} \cdot \mathrm{s}^{-1}\right)$ during top speed, and $447.7^{\circ} \cdot \mathrm{s}^{-1}\left( \pm 255^{\circ} \cdot \mathrm{s}^{-1}\right)$ and $-430.3^{\circ} \cdot \mathrm{s}^{-1}( \pm 189$ ${ }^{\circ} \cdot \mathrm{s}^{-1}$ ) during deceleration.

Furthermore, knee angles ranged between $73.5^{\circ}\left( \pm 12^{\circ}\right)$ maximal flexion and $162.6^{\circ}\left( \pm 7^{\circ}\right)$ minimal flexion during acceleration, $62.8^{\circ}\left( \pm 12^{\circ}\right)$ maximal flexion and $164.8^{\circ}\left( \pm 6^{\circ}\right)$ minimal flexion during top speed, and $81.1^{\circ}\left( \pm 16^{\circ}\right)$ maximal flexion and $168.6^{\circ}\left( \pm 12^{\circ}\right)$ minimal flexion during deceleration. Knee angular velocity varied between $935.8^{\circ} \cdot \mathrm{s}^{-1}\left( \pm 186^{\circ} \cdot \mathrm{s}^{-1}\right)$ and $-1137.8^{\circ} \cdot \mathrm{s}^{-1}\left( \pm 214^{\circ} \cdot \mathrm{s}^{-1}\right)$ during acceleration, between 1155.9 ${ }^{\circ} \cdot \mathrm{s}^{-1}\left( \pm 200^{\circ} \cdot \mathrm{s}^{-1}\right)$ and $-1208.2^{\circ} \cdot \mathrm{s}^{-1}\left( \pm 264^{\circ} \cdot \mathrm{s}^{-1}\right)$ during top speed, and $1000.1^{\circ} \cdot \mathrm{s}^{-1}\left( \pm 282^{\circ} \cdot \mathrm{s}^{-1}\right)$ and $-1004.3^{\circ} \cdot \mathrm{s}^{-1}\left( \pm 324^{\circ} \cdot \mathrm{s}^{-1}\right)$. Figure 3 illustrates continuous kinematic data of one trial of the linear $30-\mathrm{m}$ sprint test, whereas Figure 4 and Figure 5 illustrate kinematic data of one stride cycle during acceleration, top speed and deceleration of one trial. 

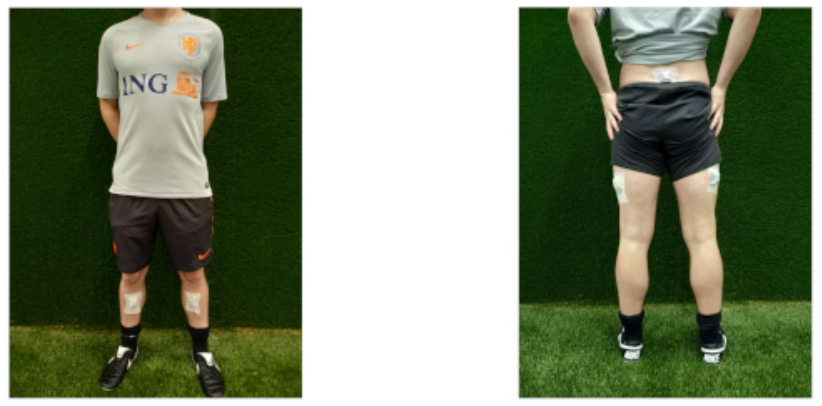

\section{A}

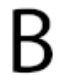

Figure 1: Representation of sensor placement. (A) Sensor placement on the right and left shank. (B) Sensor placement on the pelvis, and right and left thigh. Please click here to view a larger version of this figure.

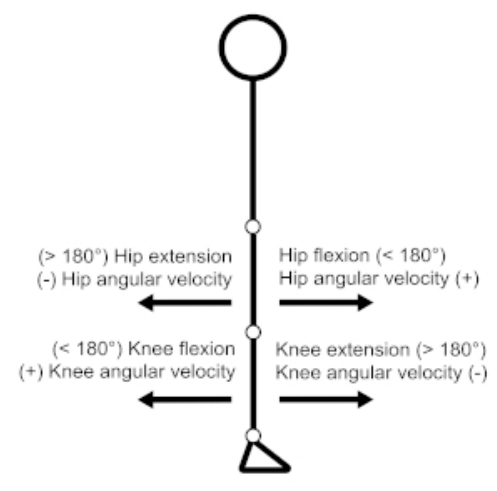

A

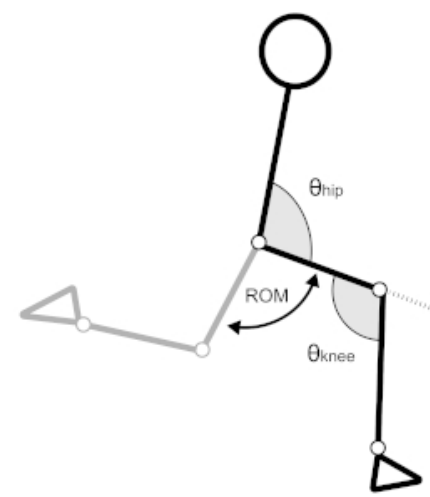

B

Figure 2: Definitions for hip and knee joint angles and angular velocities. (A) Representation of the neutral position in the sagittal plane. Joint angles in neutral position are $180^{\circ}$. (B) Representation of hip joint ( $\theta$ hip), knee joint ( $\theta$ knee) and range of motion (ROM). Please click here to view a larger version of this figure.

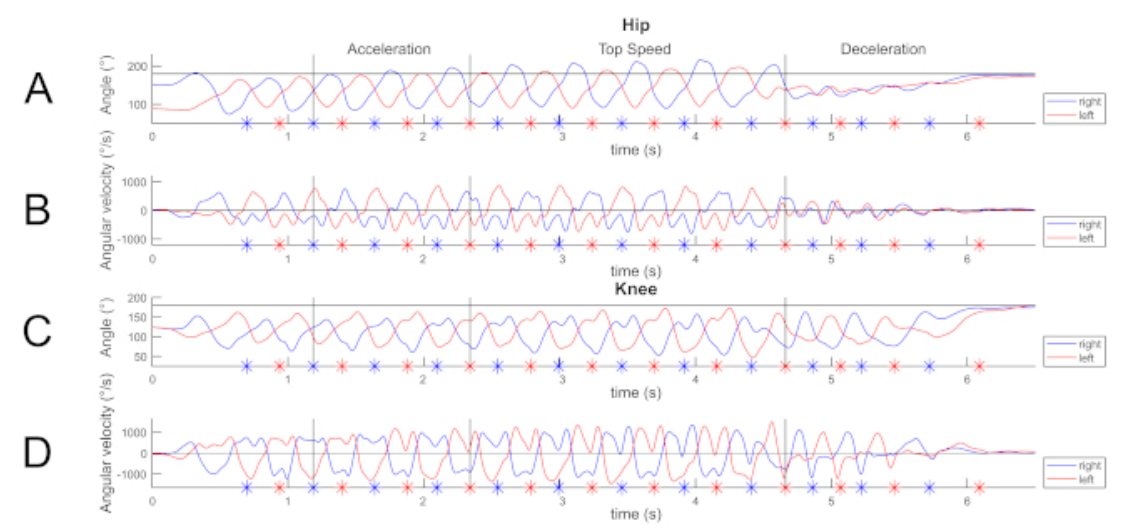

Figure 3: Visualization of sprint kinematics of one trial during accelerating, top speed and decelerating phase. An asterix indicates when a step has been detected. (A) Left and right hip flexion and extension angles over time. (B) Left and right hip angular velocities over time. (C) Left and right knee angles over time. (D) Left and right knee angular velocities over time. Please click here to view a larger version of this figure. 

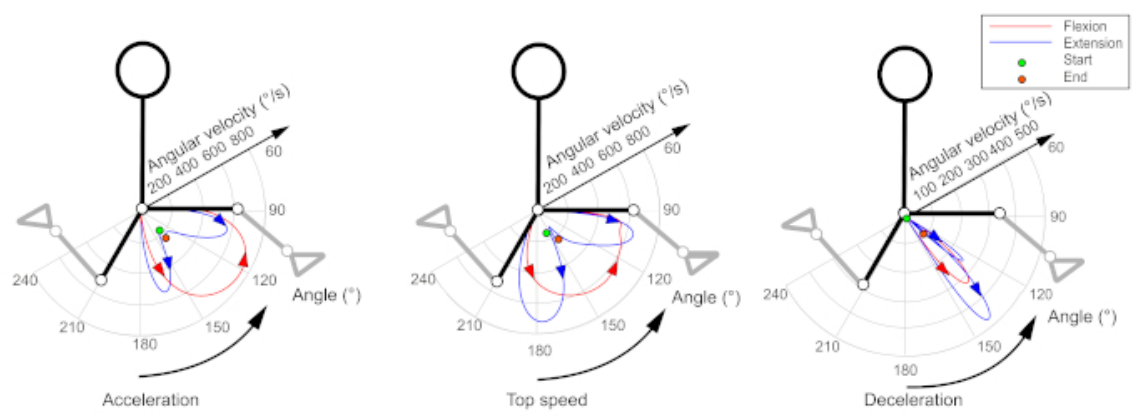

Figure 4: Polar plot in which hip joint angle $\left({ }^{\circ}\right)$ and angular velocities (flexion/extension) of one step are illustrated during acceleration, top speed and deceleration. Please click here to view a larger version of this figure.

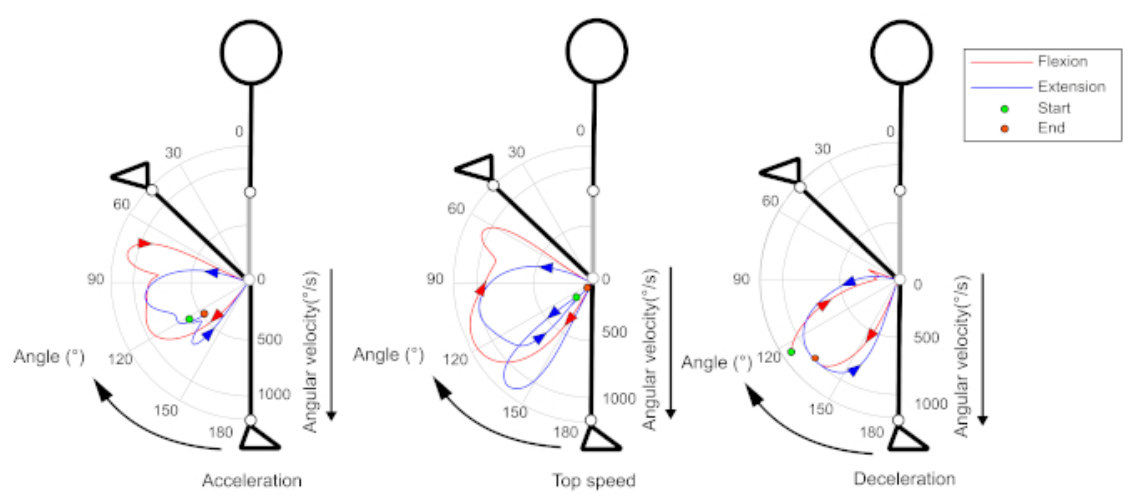

Figure 5: Polar plot in which knee joint angle $\left(^{\circ}\right)$ and angular velocities (flexion/extension) of one step are illustrated during acceleration, top speed and deceleration. Please click here to view a larger version of this figure.

\section{Discussion}

Current methods to monitor athletes in team sports do not register lower extremity kinematics, which could be a useful measure for identifying injury-risk factors. The gold standard for analysing lower extremity kinematics during sprinting are optoelectronic measurement systems ${ }^{29,30,31,32}$. Although optoelectronic measurement systems serve as a gold standard, these systems lack ecological validity due to their restricted measurement area. The sensor setup presented in this article overcomes the limitations of the current measurement systems, and is relatively cheap. The possibility to register lower extremity kinematics in the field, measured by the sensor setup, may improve athlete monitoring practice.

Previous studies that examined sprint kinematics ${ }^{29,31,37,38,39}$ reported hip angles ranging from $210^{\circ}$ extension to $90^{\circ}$ flexion. Furthermore, these studies reported knee angles ranging from $160^{\circ}$ minimal flexion and $40^{\circ}$ maximal flexion. The values observed in this study are within the range previously reported. One study ${ }^{38}$ reported hip angular velocities ranging from $-590^{\circ} \cdot \mathrm{s}^{-1}$ to $700^{\circ} \cdot \mathrm{s}^{-1}$ and knee angular velocities ranging from $-1,000^{\circ} \cdot \mathrm{s}^{-1}$ to $1,100^{\circ} \cdot \mathrm{s}^{-1}$. Although the values observed in this study were higher, they show a similar trend over time. The method has been validated and could be used for athlete monitoring in the field ${ }^{40}$.

The current study has some limitations that need to be addressed. First, apart from the characteristics of the IMUs that have been used, users must be aware that the signals derived from the IMUs are affected by several sources of error which limit the possible range of applications ${ }^{41}$. First, the oscillation of the soft tissues around the bones (i.e., soft tissue artefacts ${ }^{42}$ ) can affect the registration of kinematics. For this reason, it is important to carefully attach the IMUs to the subject's body according to the steps described in the protocol. Although necessary steps were undertaken, it should be noted that the current study did not include extra elastic straps to prevent erroneous sensor movement. This could improve results and may be seen as a limitation of this study. Second, ferromagnetic disturbances from other devices (mainly inside buildings) change the magnitude or direction of the measured magnetic field vector of the IMU's magnetometer, thus causing errors in estimated orientation $^{43}$. Therefore, sources of ferromagnetic disturbance should be avoided as much as possible. Moreover, it must be noted that the sensor setup is not applicable to sliding tackles since the sensors will detach from the skin as a result of making contact to the ground surface. Thus, participants should be instructed not to perform sliding tackles during small sided games. A possible solution for this problem could be to integrate the sensor setup in smart garments (i.e., a Smart Sensor Tights).

The kinematic variables obtained by the sensor setup could be used in a segmental model in order to monitor athletes in the field. Previous research found reduced maximum combined hip flexion and knee extension angle (i.e., theoretical hamstring length) after each half of a soccer match simulation ${ }^{44}$. In the same study, an increase in shank angular velocity has been observed during the ends of each half. The lower hamstring length combined with an increased shank velocity may indicate an increased risk of excessive hamstring strain after fatigue. Such alterations in sprinting kinematics may be detected in a field setting using an inertial measurement unit (IMU) driven segmental model. Besides changes in joint kinematics, forces that act on the body as a whole can be estimated as well. Ground reaction forces (GRF) describe the biomechanical loading experienced by the total musculoskeletal system, and can be estimated using Newton's second law of motion (i.e., $F$ $=\mathrm{m} \cdot \mathrm{a}$ ). Current research in running used GRF estimation to optimize sprint performance ${ }^{45,46}$ or assessing potential injury risk ${ }^{47,48,49,50}$. These studies suggest that loading rates, vertical impact force peaks and horizontal breaking force are related to musculoskeletal overuse injuries. 
Although it is a challenge to estimate GRF accurately during highly dynamic team-sport specific movements ${ }^{51,52}$, the possibility to monitor these variables during measurements on the field could provide new information to optimize performance, or to prevent injuries.

The results presented in this paper are limited to monitoring lower extremity kinematics during a linear sprint, focussing on hamstring strain injury mechanism. However, it should be noted that hip and groin injuries also occur frequently in team sports ${ }^{14,17,53,54,55}$. These injuries are probably caused by repetitive involvement of kicking and change of direction. Thus, future research should not only limit their focus on sprinting in relationship with the hamstring strain injury mechanism but also focus on expanding knowledge regarding change of direction tasks ${ }^{56}$ and kicking $^{57,58,59}$ in relationship with hip and groin injuries.

To conclude, this sensor setup could be integrated in smart garments. Smart garments may enable to register lower extremity kinematics in the field during team sport specific tasks, which could improve monitoring athletes in the future. This may help professionals in a daily sports setting to evaluate their training programs and optimize them, aiming to reduce injury risk.

\section{Disclosures}

The authors have nothing to disclose.

\section{Acknowledgments}

The authors would like to thankfully acknowledge the funding sources provided by the Dutch national research organization (NWO).

Furthermore, the authors would like to thankfully acknowledge the Dutch Royal Football Association (KNVB) for facilitating the research programme by giving access to their research facilities. Lastly, the authors would like to thankfully acknowledge Thijs Wiggers for his contribution to the research programme.

\section{References}

1. Bradley, P. S. et al. High-intensity running in English FA Premier League soccer matches. Journal of Sports Sciences. 27 (2), $159-168$ (2009).

2. Di Salvo, V. et al. Performance characteristics according to playing position in elite soccer. International Journal of Sports Medicine. 28 (3), 222-227 (2007).

3. Mohr, M., Krustrup, P., Bangsbo, J. Match performance of high-standard soccer players with special reference to development of fatigue. Journal of Sports Sciences. 21 (7), 519-528 (2003).

4. Rampinini, E., Coutts, A. J., Castagna, C., Sassi, R., Impellizzeri, F. M. Variation in top level soccer match performance. International Journal of Sports Medicine. 28 (12), 1018-1024 (2007).

5. McGuinness, A., Malone, S., Hughes, B., Collins, K., Passmore, D. Physical Activity and Physiological Profiles of Elite International Female Field Hockey Players Across the Quarters of Competitive Match Play. Journal of Strength and Conditioning Research. 33 (9), $2513-2522$ (2019).

6. Ihsan, M. et al. Running Demands and Activity Profile of the New Four-Quarter Match Format in Men's Field Hockey. Journal of Strength and Conditioning Research. (2018).

7. Wallace, J. L., Norton, K. I. Evolution of World Cup soccer final games 1966-2010: game structure, speed and play patterns. Journal of Science and Medicine in Sport. 17 (2), 223-228 (2014).

8. Barnes, C., Archer, D. T., Hogg, B., Bush, M., Bradley, P. S. The Evolution of Physical and Technical Performance Parameters in the English Premier League. International Journal of Sports Medicine. 35 (13), 1095-1100 (2014).

9. Smith, D. J. A framework for understanding the training process leading to elite performance. Sports Medicine. 33 (15), $1103-1126$ (2003).

10. Soligard, T. et al. How much is too much? (Part 1) International Olympic Committee consensus statement on load in sport and risk of injury. British Journal of Sports Medicine. 50 (17), 1030-1041 (2016).

11. Jaspers, A., Brink, M. S., Probst, S. G. M., Frencken, W. G. P., Helsen, W. F. Relationships Between Training Load Indicators and Training Outcomes in Professional Soccer. Sports Medicine. 47 (3), 533-544 (2017).

12. van der Horst, N., Smits, D. W., Petersen, J., Goedhart, E. A., Backx, F. J. The preventive effect of the nordic hamstring exercise on hamstring injuries in amateur soccer players: a randomized controlled trial. American Journal of Sports Medicine. 43 (6), 1316-1323 (2015).

13. van de Hoef, P. A. et al. Does a bounding exercise program prevent hamstring injuries in adult male soccer players? - A cluster-RCT. Scandinavian Journal of Medicine \& Science in Sports. 29 (4), 515-523 (2019).

14. Ekstrand, J., Hagglund, M., Walden, M. Epidemiology of muscle injuries in professional football (soccer). American Journal of Sports Medicine. 39 (6), 1226-1232 (2011).

15. Woods, C. et al. The Football Association Medical Research Programme: an audit of injuries in professional football - analysis of hamstring injuries. British Journal of Sports Medicine. 38 (1), 36-41 (2004).

16. Barboza, S. D., Joseph, C., Nauta, J., van Mechelen, W., Verhagen, E. Injuries in Field Hockey Players: A Systematic Review. Sports Medicine. 48 (4), 849-866 (2018).

17. Delfino Barboza, S., Nauta, J., van der Pols, M. J., van Mechelen, W., Verhagen, E. A. L. M. Injuries in Dutch elite field hockey players: A prospective cohort study. Scandinavian Journal of Medicine \& Science in Sports. 28 (6), 1708-1714 (2018).

18. Jones, A. et al. Epidemiology of injury in English Professional Football players: A cohort study. Physical Therapy in Sport. 35 18-22 (2019).

19. Ekstrand, J., Walden, M., Hagglund, M. Hamstring injuries have increased by 4\% annually in men's professional football, since 2001 : a 13year longitudinal analysis of the UEFA Elite Club injury study. British Journal of Sports Medicine. 50 (12), 731-737 (2016).

20. Thorborg, K. et al. Effect of specific exercise-based football injury prevention programmes on the overall injury rate in football: a systematic review and meta-analysis of the FIFA 11 and 11+ programmes. British Journal of Sports Medicine. 51 (7), $562-571$ (2017).

21. Shield, A. J., Bourne, M. N. Hamstring Injury Prevention Practices in Elite Sport: Evidence for Eccentric Strength vs. Lumbo-Pelvic Training Sports Medicine. 48 (3), 513-524 (2018). 
22. Ekstrand, J. Keeping your top players on the pitch: the key to football medicine at a professional level. British Journal of Sports Medicine. 47 (12), 723-724 (2013).

23. Hagglund, M. et al. Injuries affect team performance negatively in professional football: an 11-year follow-up of the UEFA Champions League injury study. British Journal of Sports Medicine. 47 (12), 738-742 (2013).

24. Akenhead, R., Nassis, G. P. Training Load and Player Monitoring in High-Level Football: Current Practice and Perceptions. International Journal of Sports Physiology and Performance. 11 (5), 587-593 (2016).

25. Vanrenterghem, J., Nedergaard, N. J., Robinson, M. A., Drust, B. Training Load Monitoring in Team Sports: A Novel Framework Separating Physiological and Biomechanical Load-Adaptation Pathways. Sports Medicine. 47 (11), 2135-2142 (2017).

26. Boyd, L. J., Ball, K., Aughey, R. J. The reliability of MinimaxX accelerometers for measuring physical activity in Australian football. International Journal of Sports Physiology and Performance. 6 (3), 311-321 (2011).

27. Barrett, S., Midgley, A., Lovell, R. PlayerLoad: reliability, convergent validity, and influence of unit position during treadmill running International Journal of Sports Physiology and Performance. 9 (6), 945-952 (2014).

28. Ehrmann, F. E., Duncan, C. S., Sindhusake, D., Franzsen, W. N., Greene, D. A. GPS and Injury Prevention in Professional Soccer. Journal of Strength and Conditioning Research. 30 (2), 360-367 (2016).

29. Chumanov, E. S., Heiderscheit, B. C., Thelen, D. G. The effect of speed and influence of individual muscles on hamstring mechanics during the swing phase of sprinting. Journal of Biomechanics. 40 (16), 3555-3562 (2007).

30. Heiderscheit, B. C. et al. Identifying the time of occurrence of a hamstring strain injury during treadmill running: a case study. Clinical Biomechanics. 20 (10), 1072-1078 (2005).

31. Thelen, D. G. et al. Hamstring muscle kinematics during treadmill sprinting. Medicine \& Science in Sports \& Exercise. 37 (1), $108-114$ (2005).

32. Schache, A. G., Wrigley, T. V., Baker, R., Pandy, M. G. Biomechanical response to hamstring muscle strain injury. Gait \& Posture. 29 (2), 332-338 (2009).

33. Roetenberg, D., Luinge, H., Slycke, P. Xsens MVN: Full 6DOF human motion tracking using miniature inertial sensors. Xsens Motion Technologies B.V. Enschede. 1-7 (2009).

34. Roetenberg, D., Slycke, P. J., Veltink, P. H. Ambulatory position and orientation tracking fusing magnetic and inertial sensing. IEEE Transactions on Biomedical Engineering. 54 (5), 883-890 (2007).

35. Madgwick, S. O., Harrison, A. J., Vaidyanathan, A. Estimation of IMU and MARG orientation using a gradient descent algorithm. Proceedings of IEEE International Conference on Rehabilitation Robotics. Zurich, Switzerland (2011).

36. Diebel, J. Representing Attitude: Euler Angles, Unit Quaternions, and Rotation Vectors. Matrix. 58 (15-16), 1-35 (2006).

37. Struzik, A. et al. Relationship between Lower Limb Angular Kinematic Variables and the Effectiveness of Sprinting during the Acceleration Phase. Applied Bionics and Biomechanics. 20169 (2016).

38. Struzik, A. et al. Relationship between lower limbs kinematic variables and effectiveness of sprint during maximum velocity phase. Acta of Bioengineering and Biomechanics. 17 (4), 131-138 (2015).

39. Higashihara, A., Nagano, Y., Ono, T., Fukubayashi, T. Differences in hamstring activation characteristics between the acceleration and maximum-speed phases of sprinting. Journal of Sports Sciences. 36 (12), 1313-1318 (2018).

40. Wilmes, E. et al. Inertial Sensor-Based Motion Tracking in Football with Movement Intensity Quantification. Sensors (Basel). 20 (9), (2020).

41. Camomilla, V., Bergamini, E., Fantozzi, S., Vannozzi, G. Trends Supporting the In-Field Use of Wearable Inertial Sensors for Sport Performance Evaluation: A Systematic Review. Sensors. 18 (3), 873 (2018).

42. Camomilla, V., Dumas, R., Cappozzo, A. Human movement analysis: The soft tissue artefact issue. Journal of Biomechanics. 62, 1-4 (2017).

43. Robert-Lachaine, X., Mecheri, H., Larue, C., Plamondon, A. Effect of local magnetic field disturbances on inertial measurement units accuracy. Applied Ergonomics. 63, 123-132 (2017).

44. Small, K., McNaughton, L. R., Greig, M., Lohkamp, M., Lovell, R. Soccer fatigue, sprinting and hamstring injury risk. International Journal of Sports Medicine. 30 (8), 573-578 (2009).

45. Wdowski, M. M., Gittoes, M. J. R. First-stance phase force contributions to acceleration sprint performance in semi-professional soccer players. European Journal of Sport Science. 1-23 (2019).

46. Bezodis, N. E., North, J. S., Razavet, J. L. Alterations to the orientation of the ground reaction force vector affect sprint acceleration performance in team sports athletes. Journal of Sports Sciences. 35 (18), 1-8 (2017).

47. Hreljac, A. Impact and overuse injuries in runners. Medicine \& Science in Sports \& Exercise. 36 (5), $845-849$ (2004).

48. Willy, R. W. R. In-field gait retraining and mobile monitoring to address running biomechanics associated with tibial stress fracture. Scandinavian Journal of Medicine \& Science in Sports. 26 (2), 197-205 (2016).

49. van der Worp, H., Vrielink, J. W., Bredeweg, S. W. Do runners who suffer injuries have higher vertical ground reaction forces than those who remain injury-free? A systematic review and meta-analysis. British Journal of Sports Medicine. 50 (8), $450-457$ (2016).

50. Napier, C. C. Kinetic risk factors of running-related injuries in female recreational runners. Scandinavian Journal of Medicine \& Science in Sports. 28 (10), 2164-2172. (2018).

51. Wundersitz, D. W., Netto, K. J., Aisbett, B., Gastin, P. B. Validity of an upper-body-mounted accelerometer to measure peak vertical and resultant force during running and change-of-direction tasks. Sports Biomechanics. 12 (4), 403-412 (2013).

52. Nedergaard, N. J. et al. The Relationship Between Whole-Body External Loading and Body-Worn Accelerometry During Team-Sport Movements. International Journal of Sports Physiology and Performance. 12 (1), 18-26 (2017).

53. Lundgardh, F., Svensson, K., Alricsson, M. Epidemiology of hip and groin injuries in Swedish male first football league. Knee Surgery, Sports Traumatology, Arthroscopy. 1-8 (2019).

54. Werner, J., Hagglund, M., Ekstrand, J., Walden, M. Hip and groin time-loss injuries decreased slightly but injury burden remained constant in men's professional football: the 15-year prospective UEFA Elite Club Injury Study. British Journal of Sports Medicine. 53 (9), $539-546$ (2019).

55. Werner, J., Hagglund, M., Walden, M., Ekstrand, J. UEFA injury study: a prospective study of hip and groin injuries in professional football over seven consecutive seasons. British Journal of Sports Medicine. 43 (13), 1036-1040 (2009).

56. Havens, K. L., Sigward, S. M. Whole body mechanics differ among running and cutting maneuvers in skilled athletes. Gait \& Posture. 42 (3), 240-245 (2015).

57. Charnock, B. L., Lewis, C. L., Garrett, W. E., Jr., Queen, R. M. Adductor longus mechanics during the maximal effort soccer kick. Sports Biomechanics. 8 (3), 223-234 (2009).

58. Nunome, H., Inoue, K., Watanabe, K., Iga, T., Akima, H. Dynamics of submaximal effort soccer instep kicking. Journal of Sports Sciences. 36 (22), 2588-2595 (2018). 
59. Kellis, E., Katis, A., Vrabas, I. S. Effects of an intermittent exercise fatigue protocol on biomechanics of soccer kick performance. Scandinavian Journal of Medicine \& Science in Sports. 16 (5), 334-344 (2006). 